\title{
Advanced Imaging Methods
}

\author{
M.A. Sutton • F. Hild • H. Jin • X. Li • M.M. Grédiac
}

Published online: 26 February 2011

(C) Society for Experimental Mechanics 2011

The growth and development of imaging technology, coupled with extraordinary advances in computational and image processing technology, are culminating in a wide range of vision-based, non-contacting measurement methods. For components undergoing both quasi-static and dynamic loading conditions, imaging methods have been developed to make accurate measurements for (a) two-dimensional motion in nominally planar surfaces, (b) three-dimensional shape and motion measurements for curved or planar specimens and (c) volumetric measurements of shape and motion for specimens with sufficient image features. Even more remarkably, the measurements are being made over spatial

\footnotetext{
M.A. Sutton ( $\square$, SEM fellow)

Department of Mechanical Engineering,

University of South Carolina,

300 Main Street, Room A129,

Columbia, SC 29208, USA

e-mail: sutton@sc.edu

F. Hild (SEM member)

ENS Cachan,

LMT Cachan, 61 Avenue du Président Wilson,

Cachan Cedex 94235, France

H. Jin (SEM member)

Sandia National Laboratories,

Microsystems and Mechanics Materials,

7011 East Ave, MS 9409,

Livermore, CA 94551, USA

X. Li

Department of Mechanical Engineering,

University of South Carolina,

300 Main Street,

Columbia, SC 29208, USA

M.M. Grédiac (SEM member)

Universite' Blaise Pascal-IFMA,

Laboratoiré de Mecanique et Ingenieries,

Campus des Cézeaux, BP 275,

Aubiére Cedex 63175, France
}

domains ranging from meters in size to microns, and with temporal resolution that range from a few hours to nanoseconds. The broad range of instrumentation being employed to make these measurements is breathtaking; Atomic Force Microscopes, Scanning Electron Microscopes, Confocal Imaging Microscopes, Optical Microscopes, Computed Tomography Systems, Magnetic Resonance Imaging Sytems, Mega-pixel Digital Cameras, Ultra-High Speed Optical Cameras, Optical Coherence Tomographic Systems and Photo-Interferometric Systems are a few of the instruments being employed.

In recognition of the burgeoning growth of noncontacting measurement methods, nearly 100 scientists and engineers from Asia, the EU, North America and Mexico attended an international Workshop and Symposium on Advanced Imaging Methods to present their recent developments and discuss future trends. The Workshop and Symposium was held in Columbia, South Carolina from October 5-7, 2008, and was sponsored by the National Science Foundation, the Air Force Office of Scientific Research, the State of South Carolina, the University of South Carolina and industrial partners including Correlated Solutions, Inc., DRS-Hadland and Trilion. An outcome of this meeting was the development of this special issue, comprised of many of the high quality scientific studies presented at the conference. Contributors to this special issue include individuals from national laboratories, universities and industries in the US, UK, France and Asia.

The articles in the special issue are arranged so that similar aspects are together.

- Theoretical and experimental studies regarding visionbased measurement errors

- Volumetric imaging, including image correlation with CT and X-Ray images as well as optical coherence tomography

- High rate measurements coupled with modeling 
- Multiscale, microscale and nanoscale measurements

- Vision-based studies in metal forming, shape memory alloys and single crystals

Two of the articles focused on fundamentals in the stereo-vision method. The first article by Sutton, Wang, Ke, Schreier, Reu and Miller presents the basic theory for stereo-vision and outlines the way in which the theory is used to determine both bias and variability in the 3D measurement. The authors then employ the theoretical concepts to construct analytical solutions for simple examples, demonstrating excellent agreement between the theoretical predictions and computations. The companion article by Ke, Schreier, Sutton and Wang presents results from an experimental study focused on demonstrating the efficacy of the theoretical developments for the special case of negligible motion of the specimen. By comparing the theoretical results with the measurements, the authors demonstrated that the theoretical results for variability and bias are in remarkable agreement for all three components of motion. A third article in the first group, authored by Philip Reu, outlines an effective approach for estimating bias and variability when performing image matching. As noted by Reu, a straight-forward approach for estimating errors employs Fourier shifting of high resolution images of the actual pattern (e.g. $4096 \times 4096)$ and binning of images (down-sampling) to obtain reliable estimates for the local matching bias that would be expected for sub-pixel motions. It is noted that Reu's work is also useful when performing stereo-matching.

Four of the articles focused on internal measurements of deformation during loading, though entirely different imaging approaches are used for each study. The work of Ruiz, Huntley and Coupland introduces Optical Coherence Tomography and relates OCT to several other approaches via mathematical constructs. Ruiz et al employ a recently developed mathematical framework to compare several depth-resolved displacement measurement methods. For example, the authors demonstrate that tomographic reconstruction using monochromatic single plane wave illumination can be described via a linear filtering operation with a specific transfer function and impulse response. In contrast, the work of Rae, Williamson and Addiss describes the use of X-rays to record high contrast speckle-like images of the internal region of a specimen undergoing dynamic loading. By acquiring multiple X-ray images from different directions simultaneously, the authors demonstrated that internal deformations could be quantified through cross-image matching of local subsets. For their application, the results were shown to be less informative than expected due to the highly localized deformations during impact and the relatively sparse internal pattern. Leclerc, Périé, Roux and Hild presented both theoretical and experimental results indicating that volumetric digital image correlation can be used effectively to obtain deformations down to the voxel scale while using a regularization construct that employs the equilibrium gapmethod. The authors discuss issues such as spatial resolution, correlation residuals and pattern noise on their results, with images acquired experimentally using a synchrotron source. Gates, Lambros and Heath discussed their efforts to develop efficient, accurate volumetric digital image correlation approaches that would have accuracy comparable to $2 \mathrm{D}$ image correlation. The authors show that they can achieve an accuracy of 0.20 of a voxel in each direction while processing $41 \times 41 \times 41$ cubic sub-volumes in less that $3 \mathrm{~s}$ when using eight processors in a parallel computing environment.

The area of high rate experimentation is discussed in two separate articles by Moulart, Pierron, Hallett and Wisnom and Pierron, Sutton and Tiwari. Moulart described the use of high speed imaging and a modified tension split Hopkinson Bar apparatus to obtain full-field deformation measurements on glass/epoxy composite specimens. By combining the measurements with the Virtual Fields Method, the authors demonstrated the potential of the approach for estimating elastic constants for high rate loading cases. Pierron and his co-authors used ultra-high speed imaging with Imacon 200 cameras to extract fullfield strain data with a temporal resolution of $10 \mu$ s during impact of a 3 point bend specimen. Using the principle of virtual work, the impact force was estimated from the shear strains and detailed analysis of the dynamic deformation process was presented.

A series of five articles focused on the use of imaging to understand deformation behavior at reduced length scales. Jin, Haldar, Bruck and Lu employed both grid method and digital image correlation (DIC) techniques at the microscale to quantify the displacement field in the vicinity of discontinuities in the measurements (e.g., crack tips). Results from their studies suggest that a grid method has the potential to obtain data quite close to a discontinuity, with subset-based DIC producing smoother displacement fields in regions further from the tip. Haldar, Gheewala, Grande-Allen, Sutton and Bruck used optical imaging with digital cameras and microscopes to quantify deformations in Palmetto wood at lengths scales from millimeters to microns. Grégoire, Loh, Juster and Espinosa used an AFM to quantify the highly localized deformations and local material degradation that occurs in nacre when subjected to mechanical loading. Here, the authors used image correlation for the measurements and the AFM images to identify micro-scale structural changes (e.g. relative sliding of plate structures). By combining the local results with macro-scale measurements, the authors demonstrated how the local effects propagate into the macro-behavior. In this manner, the authors elucidated the highly localized deformation mechanisms that were activated during mechanical 
loading. Karanjgaokar, Oh and Chasiotis described their micro-scale measurements performed at elevated temperature to obtain quantitative deformation measurements using image correlation in thin metallic films. Their studies showed that resistively heating specimens introduces an uneven temperature distribution resulting in highly localized strain concentrations and attendant reductions in yield stress. $\mathrm{Xu}$, Jin, $\mathrm{Lu}$, Sutton and Li presented their experimental studies focused on identifying key parameters affecting the accuracy of nanoscale metrology when using an AFM. The authors demonstrated that highly accurate metrology can only be performed when the scan angle is held constant during imaging.

A series of articles describe the use of advanced imaging to improve understanding of three separate research topics: sheet metal forming, shape memory materials and single crystal sapphire. To improve understanding of the deformations in a thin sheet undergoing forming operations, Orteu, Bugarin, Harvent, Robert and Velay employed a multiple camera stereo-vision system. The sequence of images taken during the forming process is processed using a multi-camera calibration process and sparse bundle adjustment to demonstrate the potential of the approach. Kim and Daly employed both stereo image correlation and thermal imaging to improve understanding of the rich thermo-mechanical interactions that occur during the martensitic phase transformation process.

Xia and Mello innovated a combination of a phasemultiplied circular polariscope and a series interferometer. The authors used their specially designed compact system with a general stress-optic formulation derived from anisotropic piezo-optical constitutive relationships to quantify the response of a single crystal sapphire loading by a cylindrical indenter.

In closing, it is clear that non-contacting measurement methods continue to expand their range of applicability. There is no doubt that non-contacting measurement innovations will continue in the future as investigators employ new technological developments in ways that cannot be imagined to resolve increasingly complex engineering and scientific problem. Along the way, there will be opportunities aplenty for those that seek to push the frontiers of science through measurement advances and their application. For those who have the interest, and the motivation, it will be an interesting time for everyone in the community of scholars, and I for one look forward to the future and what it brings. 